\title{
Interactive web-based lifestyle intervention and metabolic syndrome: findings from the Red Ruby (a randomized controlled trial)
}

Leila Jahangiry ${ }^{1}$, Davoud Shojaeizadeh ${ }^{2}$, Mahdieh Abbasalizad Farhangi ${ }^{3}$, Mehdi Yaseri ${ }^{4}$, Kazem Mohammad $^{4}$, Mahdi Najafi ${ }^{5^{*}}$ and Ali Montazeri ${ }^{6,7^{*}}$

\begin{abstract}
Background: Metabolic syndrome is a growing public health problem worldwide. Several interventions have been proposed to specifically target the problem. This study evaluated the effectiveness of an interactive web-based lifestyle for management of metabolic syndrome.

Methods: This randomized controlled trial was conducted from June through August 2012 in Tehran, Iran. Participants were individuals with metabolic syndrome who had registered on the study website. Interested eligible participants were invited for a free clinic visit and clinical assessment. They were randomly assigned to the intervention $(n=80)$ or control $(n=80)$ group. The intervention group received an interactive web-based program called the Healthy Heart Profile and were followed for 6 months. The control group received general information on metabolic syndrome. Anthropometric measures, glycemic status, and lipid profile were evaluated at baseline, and at 3- and 6-month follow-up assessments. Metabolic syndrome was defined according to The National Cholesterol Education Program Adult Treatment Panel (ATP) III report except for waist circumference, which was modified to $\geq 90 \mathrm{~cm}$ for both genders for the Iranian population.
\end{abstract}

Results: In total, 1,437 individuals registered on the study website. The mean age of participants was 44.2 years $(\mathrm{SD}=10.0)$. There were no significant differences between the intervention and control groups on any baseline variable except that participants in the intervention group recorded higher levels of LDL. The results showed a decrease in metabolic syndrome in both groups. These reductions were significantly greater in the intervention group at the 3 - and 6-month follow-ups. The intervention group showed significantly greater decreases $(P<0.05)$ over the control group for, respectively, systolic blood pressure (3-month: -10 versus $-6 \mathrm{mmHg}$; 6-month: -11 versus $-8 \mathrm{mmHg}$ ), diastolic blood pressure (3-month: -10 versus $-4 \mathrm{mmHg}$; 6 -month: -11 versus $-6 \mathrm{mmHg}$ ), weight (3-month: -2 versus $-1 \mathrm{~kg}$; 6 -month: -4 versus $-1 \mathrm{~kg}$ ), body mass index (3-month: -0.5 versus $-0.2 \mathrm{~kg} / \mathrm{m}^{2}$; 6-month: -1.1 versus $-0.4 \mathrm{~kg} / \mathrm{m}^{2}$ ) and improvement in $\mathrm{HDL}$ (3-month: 2 versus $0.64 \mathrm{mg} / \mathrm{dl} ; 6$-month: 6 versus $4 \mathrm{mg} / \mathrm{dl})$.

Conclusion: The findings suggest that the web-based interactive program was beneficial for individuals with metabolic syndrome. Comprehensive interactive web-based prevention programs are promising to help involve patients in improving management of metabolic syndrome and adopting a healthy lifestyle.

Trial registration: IRCT201111198132N1. Registered 27 May 2013.

\footnotetext{
*Correspondence: najafik@sina.tums.ac.ir; montazeri@acecr.ac.ir

${ }^{5}$ Tehran Heart Center, Tehran University of Medical Sciences, Tehran, Iran

${ }^{6}$ Mental Health Research Group, Health Metrics Research Center, Iranian

Institutes for Health Sciences Research, ACECR, Tehran, Iran

Full list of author information is available at the end of the article
}

(C) 2015 Jahangiry et al. Open Access This article is distributed under the terms of the Creative Commons Attribution 4.0 International License (http://creativecommons.org/licenses/by/4.0/), which permits unrestricted use, distribution, and reproduction in any medium, provided you give appropriate credit to the original author(s) and the source, provide a link to the Creative Commons license, and indicate if changes were made. The Creative Commons Public Domain Dedication waiver (http://creativecommons.org/publicdomain/zero/1.0/) applies to the data made available in this article, unless otherwise stated. 


\section{Background}

Metabolic syndrome is a growing public health problem worldwide. In Iran, it affects approximately $25 \%$ of the population and has become a leading health concern because of its link to cardiovascular disease [1, 2]. Metabolic syndrome is identified by central obesity, increased triglycerides, reduced high-density lipoprotein cholesterol (HDL), hypertension, and elevated fasting blood glucose level [3]. The National Cholesterol Education Program Adult Treatment Panel (ATP) III defines metabolic syndrome as the presence of three or more of the following conditions: triglyceride level of at least $150 \mathrm{mg} / \mathrm{dl}$, HDL of $<40 \mathrm{mg} / \mathrm{dl}$ in men and $<50 \mathrm{mg} / \mathrm{dl}$ in women, systolic/diastolic blood pressure (BP) of $\geq 130$ / $85 \mathrm{~mm} \mathrm{Hg}$, fasting blood glucose (FBG) level of $\geq 110 \mathrm{mg} / \mathrm{dl}$, and waist circumference $>102 \mathrm{~cm}$ in men and $>88 \mathrm{~cm}$ in women [4]. The current definitions of central adiposity are based on data from western populations; however, a growing body of literature indicates that this cut-off likely needs to be lowered for Asian populations. Several studies in Iran have suggested that a more realistic waist circumference for Iranians is $>90 \mathrm{~cm}$ for both genders [5-7]. ATP III states that a change in lifestyle is the essential strategy for control and management for individuals at risk of cardiovascular disease [8].

Evidence shows that lifestyle intervention programs play a crucial role in the control and treatment of metabolic syndrome. Changing dietary habits and exercise behavior can bring about a healthier waistline measurement and body mass index (BMI), improve HDL and triglyceride levels, and lower BP and blood glucose levels [9]. A recent meta-analysis of results from clinical studies on lifestyle modification and metabolic syndrome showed that the proportion of people with resolved metabolic syndrome in the intervention group was approximately twofold that of the control group. The report also showed that five out of six components of metabolic syndrome decreased significantly in the intervention groups compared to the control groups [10].

Although traditional face-to-face lifestyle intervention is the gold standard for modification of lifestyle and counseling for healthy behavior [11, 12], it faces obstacles such as availability, cost, treatment, time, travel demands, and limited effectiveness [13]. Web-based lifestyle programs can reduce these obstacles while maintaining effectiveness by offering a feasible strategy to improve health status for large numbers of at-risk people either as a stand-alone approach or as a supplement to face-to-face encounters $[11,12]$. The Internet plays an increasingly important role in health care by providing a platform for large-scale delivery of information and interventions for modifying lifestyle risk factors [14].

Several review studies have explored the effectiveness of web-based and e-health intervention to improve outcomes for lifestyle modification, weight management, physical activity, and dietary intake with equivocal results [15-18]. The use of interactive web-based programs could increase the effectiveness of intervention; however, the effects of web-based interactive lifestyle modifications for people with metabolic syndrome remain unclear. The present randomized controlled trial evaluated the effectiveness of interactive web-based lifestyle intervention for metabolic syndrome. It was hypothesized that an interactive web-based educational program will promote changes in lifestyle behaviors that in turn will result in a decrease in metabolic syndrome.

\section{Methods}

\section{Design overview}

The Red Ruby was a randomized controlled trial conducted in Tehran, Iran. A detailed description of the method can be found elsewhere in a presentation of the study protocol [19]. Briefly, this study was designed to assess the effects of interactive web-based lifestyle intervention on metabolic syndrome. The main outcomes were a decrease in the components of metabolic syndrome. The study was concluded at the 6-month followup because the results indicated early effectiveness of lifestyle intervention on changing the components of metabolic syndrome $[20,21]$.

\section{Participants and inclusion criteria}

A total of 160 people diagnosed with metabolic syndrome were randomly assigned to an intervention or control group. Participants who had registered on the study website (http://www.Heartresearch.ir) from June through August 2012 were recruited. These were individuals with metabolic syndrome aged 20 years and over living in Tehran and registered on the study website. The inclusion criteria were: waist circumference $\geq 90$ (cut-off for metabolic syndrome in Iran for both genders [5-7]) and $\mathrm{BP} \geq 130 / 85$ plus one or more metabolic syndrome components, and having access to and the basic skills necessary to use the Internet. Exclusion criteria were having a history of cardiovascular disease, diabetes, cancer, or renal disease, being pregnant, taking medication for hypertension or dyslipidemia, or failing to complete the registration form. Participants were assessed at baseline and at 3- and 6-month follow-ups.

\section{Procedures}

The study procedure from enrollment to data collection and followup assessment are present in Additiinal file 1. Announcements for recruitment were placed on virtual and non-virtual environments at the Tehran Heart Center, Tehran University of Medical Sciences, and Iranian Student News Agency. These announcements offered the opportunity to take part in a free online preventive 
intervention for cardiovascular disease with the aim of promoting lifestyle behavior including a healthful diet and physical activity. Those interested in participating were invited to register and complete an online form.

The registration page included the name, gender, age, waist circumference, weight, e-mail, and address of the applicant. The homepage also explained how to measure waist circumference. The website database was reviewed by a trained research assistant to recognize registrants aged 20 and over living in Tehran (the study setting). The participants who initially enrolled were contacted by telephone for eligibility screening. During the phone interview eligible participants who were interested in participating in the study were asked to schedule a free clinic visit and clinical assessment by trained nursing staff at Tehran Heart Center.

\section{Randomization}

The enrolled participants completed all baseline assessments and were allocated to either the intervention or control group. The allocation sequence was concealed from the main investigator (LJ) in sequentially numbered, opaque, sealed and stapled envelopes. The randomization sequence was created manually by a biostatistician using Excel software to assign participants to the study arms using a 1:1 allocation ratio with a block size of 4 . Due to the nature of the study (waiting-list controlled), it was not possible to blind participants to intervention allocation.

\section{Intervention}

Participants in both the intervention and control groups were informed of their metabolic syndrome and its components by e-mail and encouraged to make appropriate changes to their dietary intake and physical activity to manage their syndrome. The participants in the intervention group received the username and password for log-in to a personal homepage and were encouraged to regularly visit their own profile.

\section{My Healthy Heart Profile}

My Healthy Heart Profile is a free Internet-based program with key interactive features designed for prevention of metabolic syndrome. My Healthy Heart Profile was structured into five parts:

1. Educational materials: This page was updated with new educational materials at least twice a month. The educational material included methods to lower cholesterol and control high blood pressure, conditions that put individuals at risk for cardiovascular disease, the need to lose weight, control of diabetes, the relationship between physical activity and the heart, and information on Dietary Approaches to Stop Hypertension (DASH).
E-mail reminders were sent to each participant each time a new educational material was posted on the website. All users received the same educational materials. The e-mail reminders contained a brief description of the educational materials and a hyperlink to the website.

2. Personal information for name, age, gender, weight, height, telephone number, and e-mail address.

3. Inbox: This was an interactive section for exchanging messages. A calorie-restricted diet tailored by a dietitian was sent to each inbox. This diet was based on each participant's calorie requirements according to his/her ideal body weight (IBW) and corrected body weight (CBW) where less than $30 \%$ of the calories were derived from fat in accordance with National Heart, Lung, and Blood Institute guidelines [22]. The inbox provided an opportunity for ongoing discussion between the user and the dietitian. The users could only communicate with the dietitian through this part of the website. Users could post their answers at any time they wished and would receive a response within 24 hours.

4. Estimation of Framingham Stroke Risk (FSR) is an interactive tool by which users can calculate cardiovascular risk for 10 years based on the Persian online version of the Framingham risk score (by permission). The FSR considers the cardiovascular risk factors of age ( $>20$ years), gender (male and female), total cholesterol (TC), HDL, systolic BP, and smoking habits. Participants can estimate their scores at every log-in and obtain feedback by text for the three traffic signals used to illustrate the three levels of risk (high, moderate, low).

5. Anthropometric and clinical measures recorded periodic measurements of user weight, waist circumference, BMI, BP, TC, LDL, HDL, triglycerides, and FBG. A simple graph displayed the measurements in one of the three warning colors (red $=$ needs attention, orange $=$ close to risk, green $=$ good) for each record.

\section{Control}

Participants in the control group were kept on a waiting list and received e-mail messages every three weeks that included information about metabolic syndrome and general information about healthy nutrition and the benefits of fruit and vegetable intake, physical activity, and lowering body weight.

\section{Outcome measures}

The primary outcomes were a change in waist circumference, weight, systolic and diastolic BP, HDL, TC, triglycerides, LDL, and FBG. Metabolic syndrome was 
defined according to The National Cholesterol Education Program ATP III as the presence of three or more of the following conditions (except for waist circumference, which was set at $\geq 90 \mathrm{~cm}$ for both genders for Iranians): triglycerides $<150 \mathrm{mg} / \mathrm{dl}, \mathrm{HDL}<40 \mathrm{mg} / \mathrm{dl}$ for men and $<50 \mathrm{mg} / \mathrm{dl}$ for women, $\mathrm{BP}<130 / 85 \mathrm{~mm}$ $\mathrm{Hg}$, and fasting blood glucose $(\mathrm{FBG})<110 \mathrm{mg} / \mathrm{dl}$.

Waist circumference was evaluated using a measuring tape to the nearest $0.1 \mathrm{~cm}$. The weight of an individual dressed in light clothing without shoes was recorded each time using a calibrated scale (Seca; model 8811021658; Germany) to the nearest $0.1 \mathrm{~kg}$. Height was measured without shoes using a stadiometer (Seca; Germany) to the nearest $0.1 \mathrm{~cm}$. BP was measured with a mercury sphygmomanometer twice in the same arm after the individual had been seated at rest for 10-15 min. The systolic and diastolic BP measurements were the mean of the two readings. Blood sampling was collected for measurement of $\mathrm{TC}$, triglycerides, LDL, HDL, and FBG for all participants. Overnight fasting for 12-14 hours was required before blood sampling. FBG was measured using the glucose oxidase method (intraand inter-assay coefficients of variation of $2.1 \%$ and $2.6 \%$, respectively). BMI was measured by using the individual's weight in kilograms divided by the square of his/her height in meters [23].

\section{Sample size}

The sample size was calculated based on a decrease in one standard deviation $(2.5 \mathrm{~cm})$ in waist circumference [24] as one of the most important components of metabolic syndrome [25]. A study with a power of $90 \%$ at $5 \%$ significance level requires 60 participants in each arm. Giving the risk of attrition risk, 80 participants per each group were sought.

\section{Statistical analysis}

The data was presented as mean, standard deviation, and percentage. Normal distribution of the data was assessed using the Kolmogorov-Smirnov test and quantile-quantile (Q-Q) plots. To compare baseline variables between two groups, the $t$-test, Mann-Whitney $\mathrm{U}$ test, chi-square, and Fisher exact tests were employed.

Any change in scores was calculated by subtracting the baseline score from the 3- and 6-month follow-up scores. Linear mixed model (LMM) analysis was used to evaluate changes within groups. Adjustment for the multiple comparisons was done using the Bonferroni method. Time points in the analyses included baseline, during intervention (3-month follow-up), and at the termination of intervention (6-month follow-up). Differences between groups for each metabolic syndrome component and total component scores were evaluated by intent-to-treat analysis using LMM. All statistical analysis was performed by STATA (version 11.0). Results were considered statistically significant at $P<0.05$.

\section{Ethics}

The ethics committee of Tehran University of Medical Sciences, Vice Chancellor for Research approved the study (number 90/130/1736). Written informed consent was obtained from all participants. The CONSORT checklist is provided in Additiinal file 2.

\section{Results}

\section{Participants}

In total, 1,437 individuals registered on the study website. Of these, 815 records were excluded because registrants were living outside Tehran $(n=356)$, had a waist circumference $<90 \mathrm{~cm}(n=392)$, or had incomplete information (no telephone number; $n=67$ ). The remaining 622 registered participants were screened for eligibility and 305 were excluded for the following morbid conditions: cardiovascular disease $(n=68)$, diabetes $(n=51)$, consumption of antihypertensive medication $(n=45)$, consumption of cholesterol-lowering medication $(n=32)$, blood pressure $<130 / 85(\mathrm{mmHg})(n=96)$, renal disease or cancer $(n=12)$, or pregnancy $(n=1)$. A total of 317 individuals were invited to participate. Of these, 229 were able to attend clinical assessments and were scheduled for a baseline visit. Of the attending participants for clinical assessments, 171 met the criteria for inclusion and had metabolic syndrome. Of these, 160 participants agreed to complete the baseline measures and were randomly assigned to intervention and control groups (see Additional file 1).

The characteristics of the participants are presented in Table 1. The mean age of participants was 44.2 years $(\mathrm{SD}=10.0)$ and ranged from 21 to 68 years. Participants were predominantly male (66.3\%) and married (83.8 \%). Mean (SD) Internet usage was 13.2 hours per week $(\mathrm{SD}=15.5)$. The mean weight was $87 \mathrm{~kg}(\mathrm{SD}=15)$ and BMI was $30.1 \mathrm{~kg} / \mathrm{m}^{2}$ (SD = 4.6). There were no significant differences between the intervention and control groups on any baseline variable, except for LDL; participants in the intervention group showed higher LDL values than the control group.

The attrition rate in the intervention and control groups at first follow-up was the same (20 \%); however, the control group had a significantly higher attrition rate $(33.7 \%)$ compared to the intervention group $(20 \%)$ at the 6-month follow-up. This information has been discussed elsewhere [26].

\section{Changes in metabolic syndrome indicators}

Table 2 shows the distribution of baseline and follow-up values for changes in metabolic syndrome indicators over time. The results of mixed model repeated 
Table 1 Baseline characteristics of study participants

\begin{tabular}{|c|c|c|c|c|}
\hline & Total $(n=160)$ & Control $(n=80)$ & Intervention $(n=80)$ & $P$ \\
\hline Age (mean, SD) & $44.5 \pm 10$ & $44.8 \pm 10$ & $43.3 \pm 10.1$ & $0.345^{a}$ \\
\hline Gender (number, \%) & & & & $0.403^{c}$ \\
\hline Males & $106(66.3)$ & $56(70.0)$ & $50(62.5)$ & \\
\hline Females & $54(33.8)$ & $24(30.0)$ & $30(37.5)$ & \\
\hline Education (number, \%) & & & & $0.080^{c}$ \\
\hline$\leq 12$ & $71(44.4)$ & $41(51.3)$ & $30(37.5)$ & \\
\hline$>12$ & 89 (55.6) & $39(48.8)$ & $50(62.5)$ & \\
\hline Marital status (number, \%) & & & & $0.113^{\mathrm{d}}$ \\
\hline Single & $21(13.1)$ & $14(17.5)$ & $7(8.8)$ & \\
\hline Married & $134(83.8)$ & $62(77.5)$ & $72(90.0)$ & \\
\hline Divorced/widowed & $5(3.1)$ & $4(5.0)$ & $1(1.3)$ & \\
\hline Employment status (number, \%) & & & & $>0.99^{d}$ \\
\hline Employed & $159(99.4)$ & $79(98.8)$ & $80(100.0)$ & \\
\hline Unemployed & $1(0.6)$ & $1(1.3)$ & $0(0.0)$ & \\
\hline Smoking (number, \%) & $22(13.8)$ & $8(10.0)$ & $14(17.5)$ & $0.168^{c}$ \\
\hline Alcohol drinking (number, \%) & $9(5.7)$ & $5(6.3)$ & $4(5.1)$ & $>0.99^{d}$ \\
\hline Drug addiction (number, \%) & $2(1.3)$ & $2(2.5)$ & $0(0.0)$ & $0.497^{d}$ \\
\hline Internet usage (hours/week) (mean, SD) & $13.2(15.5)$ & $10.9(13.7)$ & $15.5(16.9)$ & 0.061 \\
\hline Weight (kg) (mean, SD) & $87 \pm 15$ & $88 \pm 14$ & $87 \pm 16$ & $0.574^{\mathrm{a}}$ \\
\hline Body mass index $\left(\mathrm{kg} / \mathrm{m}^{2}\right)$ (mean, SD) & $30.1 \pm 4.6$ & $30.5 \pm 4.5$ & $29.8 \pm 4.7$ & $0.374^{a}$ \\
\hline Waist circumference $(\mathrm{cm})$ (mean, SD) & $104 \pm 9$ & $105 \pm 9$ & $103 \pm 9$ & $0.199^{a}$ \\
\hline Systolic blood pressure (mmHg) (mean, SD) & $132 \pm 11$ & $132 \pm 13$ & $131 \pm 8$ & $0.593^{\mathrm{a}}$ \\
\hline Diastolic blood pressure (mmHg) (mean, SD) & $88 \pm 6$ & $88 \pm 7$ & $89 \pm 6$ & $0.594^{a}$ \\
\hline Total cholesterol (mg/dl) (mean, SD) & $195 \pm 39$ & $191 \pm 35$ & $199 \pm 44$ & $0.181^{\mathrm{a}}$ \\
\hline LDL cholesterol (mg/dl) (mean, SD) & $129 \pm 32$ & $123 \pm 31$ & $134 \pm 33$ & $0.019^{a}$ \\
\hline \multicolumn{5}{|l|}{ HDL cholesterol (mg/dl) (mean, SD) } \\
\hline Women & $39 \pm 8$ & $39 \pm 10$ & $39 \pm 7$ & $0.683^{b}$ \\
\hline Men & $46 \pm 11$ & $48 \pm 12$ & $44 \pm 10$ & $0.086^{\mathrm{b}}$ \\
\hline Triglycerides (mg/dl) (mean, SD) & $192 \pm 113$ & $198 \pm 127$ & $186 \pm 96$ & $0.534^{b}$ \\
\hline Fasting blood glucose (mg/dl) (mean, SD) & $90 \pm 14$ & $91 \pm 15$ & $89 \pm 12$ & $0.772^{b}$ \\
\hline Physical activity (met/mins.) (mean, SD) & $493 \pm 716$ & $471 \pm 750$ & $515 \pm 684$ & $0.224^{b}$ \\
\hline
\end{tabular}

${ }^{\mathrm{a}}$ Derived from $t$-test. ${ }^{\mathrm{b}}$ Derived from Mann-Whitney test. ${ }^{\mathrm{C}}$ Derived from chi-square test. ${ }^{\mathrm{d}}$ Derived from Fisher exact test

measures analysis, including the interaction between time and group, are shown. The interaction was significant for weight, BMI, systolic and diastolic BP, and HDL and indicated that the interactive web-based intervention improved metabolic syndrome components.

Participants in both the intervention and control groups had lost a significant amount of weight by the 6-month follow-up. This was significantly greater in the intervention group. At the 6-month follow-up, the intervention group showed a significantly greater decrease $(P<0.001)$ in systolic BP (3-month: -10 versus $-6 \mathrm{mmHg}$ and 6month: -11 versus $-8 \mathrm{mmHg}$, respectively) and diastolic BP (3-month: -10 versus $-4 \mathrm{mmHg}$ and 6-month: -6 versus $-11 \mathrm{mmHg})$. Significant changes in $\mathrm{HDL}$ and BMI
$(P<0.05)$ were apparent in the intervention group during follow-up. Within-group changes were observed in both the intervention and control groups for weight, BMI, waist circumference, BP, and TG during the follow-up assessments. The within-group changes were significant in the intervention group, but not in the control group.

The overall number of participants with metabolic syndrome (showing at least three components) between groups was also assessed. There were no significant differences between groups for the number of metabolic syndrome components at baseline, but generalized LMM analysis (GLMM) at follow-up showed significant differences between groups for the number of metabolic syndrome components $(P=0.007$; Fig. 1$)$. 
Table 2 Changes in cardiometabolic risk factors over time between groups

\begin{tabular}{|c|c|c|c|c|c|}
\hline & Time & & Control & Intervention & Time/group interaction ${ }^{a}$ \\
\hline \multirow[t]{7}{*}{ Weight (kg) } & Baseline & Value & $88 \pm 14$ & $87 \pm 16$ & $<0.001$ \\
\hline & 3-month follow-up & Value & $86 \pm 13$ & $85 \pm 14$ & \\
\hline & & Change & $-1 \pm 3$ & $-2 \pm 2$ & \\
\hline & & $p^{\mathrm{b}}$ & 0.059 & $<0.001$ & \\
\hline & 6-month follow-up & Value & $87 \pm 12$ & $83 \pm 15$ & \\
\hline & & Change & $-1 \pm 3$ & $-4 \pm 3$ & \\
\hline & & $P^{b}$ & $<0.001$ & $<0.001$ & \\
\hline \multirow[t]{7}{*}{ Body mass index $\left(\mathrm{kg} / \mathrm{m}^{2}\right)$} & Baseline & Value & $30.5 \pm 4.5$ & $29.8 \pm 4.7$ & 0.009 \\
\hline & 3-month follow-up & Value & $29.8 \pm 3.8$ & $29 \pm 4$ & \\
\hline & & Change & $-0.2 \pm 1$ & $-0.5 \pm 1$ & \\
\hline & & $p^{\mathrm{b}}$ & 0.079 & $<0.001$ & \\
\hline & 6-month follow-up & Value & $29.5 \pm 3.5$ & $28.6 \pm 4.4$ & \\
\hline & & Change & $-0.4 \pm 1$ & $-1.1 \pm 1$ & \\
\hline & & $p^{\mathrm{b}}$ & $<0.001$ & $<0.001$ & \\
\hline \multirow[t]{7}{*}{ Waist circumference (cm) } & Baseline & Value & $105 \pm 9$ & $103 \pm 9$ & 0.64 \\
\hline & 3-month follow-up & Value & $103 \pm 8$ & $101 \pm 8$ & \\
\hline & & Change & $-2 \pm 4$ & $-3 \pm 3$ & \\
\hline & & $p^{\mathrm{b}}$ & 0.385 & $<0.001$ & \\
\hline & 6-month follow-up & Value & $100 \pm 18$ & $99 \pm 9$ & \\
\hline & & Change & $-5 \pm 19$ & $-4 \pm 3$ & \\
\hline & & $p^{b}$ & 0.012 & $<0.001$ & \\
\hline \multirow[t]{7}{*}{ Systolic blood pressure (mmHg) } & Baseline & Value & $132 \pm 13$ & $131 \pm 8$ & 0.016 \\
\hline & 3-month follow-up & Value & $127 \pm 11$ & $121 \pm 13$ & \\
\hline & & Change & $-6 \pm 14$ & $-10 \pm 11$ & \\
\hline & & $p^{\mathrm{b}}$ & $<0.001$ & $<0.001$ & \\
\hline & 6-month follow-up & Value & $124 \pm 8$ & $120 \pm 10$ & \\
\hline & & Change & $-8 \pm 14$ & $-11 \pm 9$ & \\
\hline & & $p^{b}$ & $<0.001$ & $<0.001$ & \\
\hline \multirow[t]{7}{*}{ Diastolic blood pressure $(\mathrm{mmHg})$} & Baseline & Value & $88 \pm 7$ & $89 \pm 6$ & 0.006 \\
\hline & 3-month follow-up & Value & $84 \pm 9$ & $80 \pm 9$ & \\
\hline & & Change & $-4 \pm 8$ & $-10 \pm 9$ & \\
\hline & & $p^{\mathrm{b}}$ & $<0.001$ & $<0.001$ & \\
\hline & 6-month follow-up & Value & $82 \pm 6$ & $78 \pm 6$ & \\
\hline & & Change & $-6 \pm 7$ & $-11 \pm 6$ & \\
\hline & & $p^{b}$ & $<0.001$ & $<0.001$ & \\
\hline \multirow[t]{7}{*}{ Fasting blood glucose (mg/dl) } & Baseline & Value & $91 \pm 15$ & $89 \pm 12$ & 0.627 \\
\hline & 3-month follow-up & Value & $89 \pm 13$ & $85 \pm 10$ & \\
\hline & & Change & $-1 \pm 12$ & $-4 \pm 12$ & \\
\hline & & $P^{\mathrm{b}}$ & 0.778 & 0.004 & \\
\hline & 6-month follow-up & Value & $88 \pm 13$ & $85 \pm 8$ & \\
\hline & & Change & $-2 \pm 14$ & $-4 \pm 12$ & \\
\hline & & $p^{\mathrm{b}}$ & 0.39 & 0.028 & \\
\hline
\end{tabular}


Table 2 Changes in cardiometabolic risk factors over time between groups (Continued)

\begin{tabular}{|c|c|c|c|c|c|}
\hline \multirow[t]{7}{*}{ Total cholesterol (mg/dl) } & Baseline & Value & $191 \pm 35$ & $199 \pm 44$ & 0.25 \\
\hline & 3 months follow-up & Value & $188 \pm 34$ & $186 \pm 37$ & \\
\hline & & Change & $-4 \pm 29$ & $-13 \pm 45$ & \\
\hline & & $p^{\mathrm{b}}$ & 0.409 & 0.019 & \\
\hline & 6-month follow-up & Value & $186 \pm 28$ & $186 \pm 34$ & \\
\hline & & Change & $-6 \pm 24$ & $-13 \pm 41$ & \\
\hline & & $P^{\mathrm{b}}$ & 0.591 & 0.049 & \\
\hline \multirow[t]{7}{*}{ Triglycerides (mg/dl) } & Baseline & Value & $198 \pm 127$ & $186 \pm 96$ & 0.606 \\
\hline & 3-month follow-up & Value & $175 \pm 161$ & $160 \pm 84$ & \\
\hline & & Change & $-29 \pm 132$ & $-18 \pm 85$ & \\
\hline & & $P^{\mathrm{b}}$ & 0.075 & 0.04 & \\
\hline & 6-month follow-up & Value & $144 \pm 68$ & $129 \pm 54$ & \\
\hline & & Change & $-53 \pm 82$ & $-51 \pm 77$ & \\
\hline & & $p^{\mathrm{b}}$ & 0.029 & $<0.001$ & \\
\hline \multirow[t]{8}{*}{$\mathrm{HDL}(\mathrm{mg} / \mathrm{dl})$} & Baseline & Value & $42 \pm 11$ & $41 \pm 8$ & 0.018 \\
\hline & & & $0 \pm 0$ & $0 \pm 0$ & \\
\hline & 3-month follow-up & Value & $42 \pm 12$ & $42 \pm 9$ & \\
\hline & & Change & $0.64 \pm 11$ & $2 \pm 9$ & \\
\hline & & $P^{b}$ & 0.115 & 0.066 & \\
\hline & 6-month follow-up & Value & $44 \pm 11$ & $46 \pm 7$ & \\
\hline & & Change & $4 \pm 11$ & $6 \pm 8$ & \\
\hline & & $p^{\mathrm{b}}$ & 0.066 & $<0.001$ & \\
\hline \multirow[t]{7}{*}{ LDL } & Baseline & Value & $123 \pm 31$ & $134 \pm 33$ & 0.412 \\
\hline & 3-month follow-up & Value & $118 \pm 29$ & $126 \pm 33$ & \\
\hline & & Change & $-6 \pm 25$ & $-6 \pm 30$ & \\
\hline & & $P^{\mathrm{b}}$ & 0.062 & 0.089 & \\
\hline & 6-month follow-up & Value & $121 \pm 22$ & $126 \pm 27$ & \\
\hline & & Change & $-3 \pm 22$ & $-7 \pm 31$ & \\
\hline & & $P^{\mathrm{b}}$ & 0.089 & 0.124 & \\
\hline
\end{tabular}

Based on mixed model

${ }^{\mathrm{b}}$ Based on mixed model, adjusted for the multiple comparisons by Bonferroni method

\section{Discussion}

The findings from this web-based interactive randomized controlled trial showed that 6 months of lifestyle intervention positively influenced several metabolic syndrome components. The number of participants with metabolic syndrome decreased in both arms of the study; however, a significantly greater decrease accompanied by a lower attrition rate was observed for the intervention group. The lifestyle intervention used was an interactive education strategy that provided information and skills for participants to assist them in achieving the recommended physical activity levels and following a healthful diet plan.

Significant improvements were observed for weight loss, reduced BMI, systolic, and diastolic BP, and increased HDL at the 6-month follow-up. A similar study from Korea showed that an 8-week Internet-based cardiovascular risk reduction program resulted in significant changes in cardiovascular risk, waist circumference, diastolic blood pressure, and fasting plasma glucose among male workers with metabolic syndrome [27]. Although some studies detect no effect on cholesterol management [28], evidence from recent studies support the current findings [29-31]. Bond et al. [32] reported that a 6-month web-based intervention in addition to usual simple care increased HDL-C, and decreased weight and $\mathrm{HbA1C}$ in patients with type 2 diabetes mellitus. They reported that serum cholesterol and weight decreased in both the intervention and control groups; however, the decrease was greater in the intervention group $(P<0.05)$, results which are consistent with the findings of the present study.

Web-based interactive programs can motivate participants to adhere better to clinical assessments and 


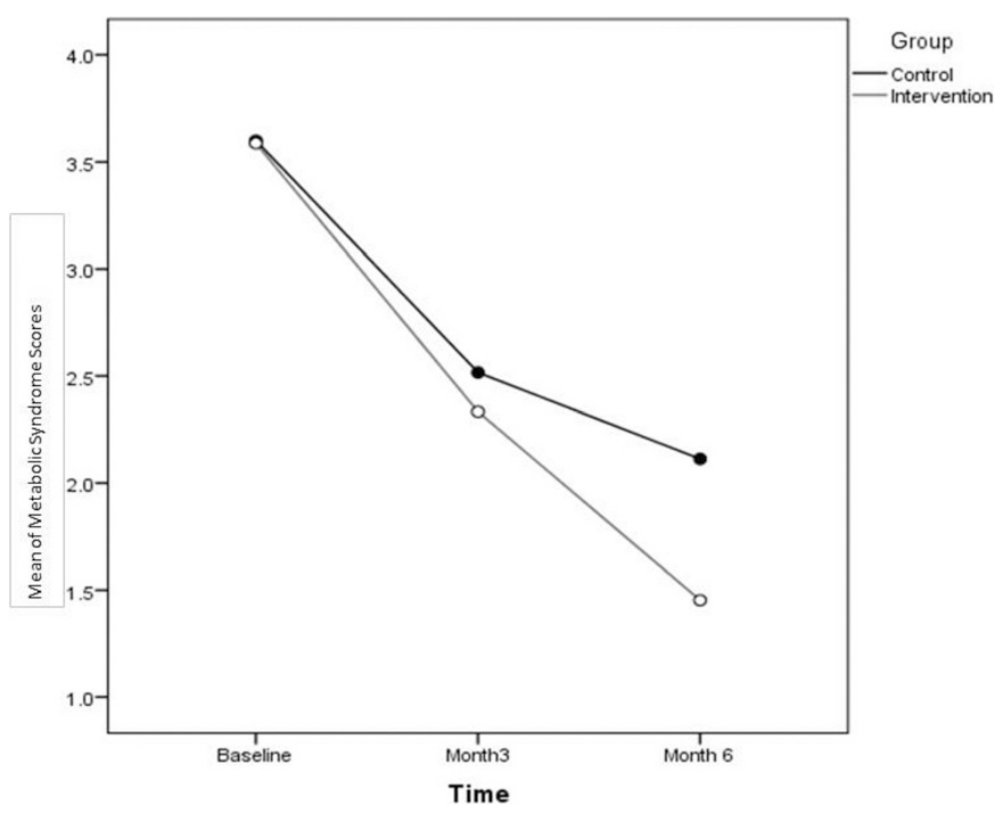

Fig. 1 Mean values for metabolic syndrome components

desired behaviors for physical activity and a healthful diet. Online health assessments provide valuable feedback and facilitate opportunistic intervention by including assessment of multiple risk factors that provides a comprehensive picture for patients and encourages them to change lifestyle risk factors [31, 33].

One consistent finding on the topic is that the use of multiple behavioral change techniques significantly increased the effectiveness of a program [34-36]. The present study showed that the use of an interactive website that is frequently updated for informational content with e-mail notifications, interactive risk assessment tools, and tracking tools appeared to contribute to a change in lifestyle and had a positive effect on metabolic syndrome components. Assessing cardiovascular disease risk by using easy-to-use online risk assessment tools could facilitate better decisionmaking about lifestyle recommendations.

Risk factor assessment is the first step in primary prevention and guides treatment strategy because the intensity of the preventive recommendations is tailored to a patient's level of risk $[37,38]$. The mode of delivery was a key influencing factor when encouraging and persuading a participant to follow clinical assessments. Similar studies have shown that sending tailored and timely feedback, providing regular new and specific content with periodic prompts, and sending reminders result in better outcomes [39]. It appears that, in the current study, following up of participants for clinical assessment, sending the results of clinical assessments by email, and recording the assessments on their profiles were additional factors to achieving successful results for metabolic syndrome management.
One surprising finding was that, although women were more likely to use the Internet to look for health information than men, in line with previous studies [40-42], men were more interested in the web-based programs and participated more than women $(66.3 \%$ versus $33.8 \%$ ). This study highlights the importance of interactive web-based programs that have shown promise for effective changes in lifestyle. However, in the battle against the epidemic of metabolic syndrome, the primary care settings are still the front lines [43].

\section{Conclusions}

This randomized controlled trial showed the potential benefits of a web-based interactive program for individuals with metabolic syndrome. The comprehensive interactive web-based prevention program was a promising method of increasing the involvement of the participants in improved management of metabolic syndrome and adoption of a healthy lifestyle.

\section{Additional files}

Additional file 1: Flow diagram of study. Flow diagram of the study based on the CONSORT statement. (DOC $35 \mathrm{~kb}$ )

Additional file 2: CONSORT checklist for study. File contains the checklist for the study as suggested by the CONSORT statement. (DOC 206 kb)

\section{Abbreviations}

ATP: Adult Treatment Panel; BMI: body mass index; BP: blood pressure; CBW: corrected body weight; CVD: cardiovascular disease; DASH: Dietary Approaches to Stop Hypertension; FFQ: food frequency questionnaire; FSR: Framingham Risk Score; GHQ: general health questionnaire; HDL: highdensity lipoproteins; IBW: ideal body weight; IPAQ: International Physical Activity Questionnaire; LDL: low-density lipoproteins; QOL: quality of life; 
$\mathrm{RCT}$ : randomized controlled trial; $\mathrm{TC}$ : total cholesterol; WC: waist circumference.

\section{Competing interests}

The authors declare that they have no competing interests.

\section{Authors' contributions}

$\sqcup J$ was the main investigator, designed the study, collected the data, and wrote the first draft. DS contributed to the writing process. MAF provided the diet plan and carried out online nutritional counseling. MY and KM performed the statistical analysis. MN participated in the design, coordination, recruitment, data collection, and clinical assessments. AM contributed to the design, coordinated the study, and provided the final manuscript. All authors read and approved the final manuscript.

\section{Acknowledgments}

This manuscript originated from a Ph.D. thesis (240\2425) by Leila Jahangiry, Department of Health Education and Promotion, School of Public Health, Tehran University of Medical Sciences, Tehran, Iran. We are grateful to the Tehran Heart Center for providing facilities for the study. We wish to thank the Iranian Institute for Health Sciences Research (ACECR) for their support for the project. This study was not funded by special institutes.

\section{Author details}

${ }^{1}$ Department of Health Education and Health Promotion, School of Public Health, Tabriz University of Medical Sciences, Tabriz, Iran. ${ }^{2}$ Department of Health Education and Health Promotion, School of Public Health, Tehran University of Medical Sciences, Tehran, Iran. ${ }^{3}$ Department of Community Nutrition, Faculty of Health and Nutrition, Tabriz University of Medical Sciences, Tabriz, Iran. ${ }^{4}$ Department of Epidemiology and Biostatistics, School of Public Health, Tehran University of Medical Sciences, Tehran, Iran. ${ }^{5}$ Tehran Heart Center, Tehran University of Medical Sciences, Tehran, Iran. ${ }^{6}$ Mental Health Research Group, Health Metrics Research Center, Iranian Institutes for Health Sciences Research, ACECR, Tehran, Iran. ${ }^{7}$ Faculty of Humanity Sciences, University of Science and Culture, ACECR, Tehran, Iran.

\section{Received: 18 February 2015 Accepted: 9 September 2015}

\section{Published online: 21 September 2015}

\section{References}

1. Esmaillzadeh A, Mirmiran P, Azadbakht L, Etemadi A, Azizi F. High prevalence of the metabolic syndrome in Iranian adolescents. Obesity (Silver Spring). 2006;14:377-82.

2. Azizi F, Salehi P, Etemadi A, Zahedi-Asl S. Prevalence of metabolic syndrome in an urban population: Tehran Lipid and Glucose Study. Diabetes Res Clin Pract. 2003;61:29-37.

3. National Cholesterol Education Program (NCEP) Expert Panel on Detection E, and Treatment of High Blood Cholesterol in Adults (Adult Treatment Panel III). Third report of the National Cholesterol Education Program (NCEP) expert panel on detection, evaluation, and treatment of high blood cholesterol in adults (Adult Treatment Panel III) final report. Circulation. 2002;106:3143-421.

4. Cleeman J, Grundy S, Becker D, Clark L. Expert panel on detection, evaluation and treatment of high blood cholesterol in adults. Executive summary of the third report of the National Cholesterol Education Program (NCEP) Adult Treatment Panel (ATP III). JAMA. 2001;285:2486-97.

5. Esteghamati A, Abbasi M, Rashidi A, Meysamie A, Khalilzadeh O, Haghazali M, et al. Optimal waist circumference cut-offs for the diagnosis of metabolic syndrome in Iranian adults: results of the third national survey of risk factors of non-communicable diseases (SuRFNCD-2007). Diabet Med. 2009;26:745-6.

6. Delavar MA, Lye MS, Khor GL, Hassan ST, Hanachi P. Dietary patterns and the metabolic syndrome in middle aged women, Babol. Iran Asia Pac J Clin Nutr. 2009;18:285-92.

7. Azizi F, Khalili D, Aghajani H, Esteghamati A, Hosseinpanah F, Delavari A, et al. Appropriate waist circumference cut-off points among Iranian adults: the first report of the Iranian National Committee of Obesity. Arch Iran Med. 2010;13:243-4.

8. Grundy SM, Cleeman Jl, Bairey Merz CN, Brewer JHB, Clark LT, Hunninghake DB, et al. Implications of recent clinical trials for the National Cholesterol Education Program Adult Treatment Panel III guidelines. J Am Coll Cardiol. 2004:44:720-32.
9. Dunkley AJ, Charles K, Gray LJ, Camosso-Stefinovic J, Davies MJ, Khunti K. Effectiveness of interventions for reducing diabetes and cardiovascular disease risk in people with metabolic syndrome: systematic review and mixed treatment comparison meta-analysis. Diabetes Obes Metab. 2012;14:616-25.

10. Yamaoka K, Tango T. Effects of lifestyle modification on metabolic syndrome: a systematic review and meta-analysis. BMC Med. 2012;10:138.

11. Digenio AG, Mancuso JP, Gerber RA, Dvorak RV. Comparison of methods for delivering a lifestyle modification program for obese patients: a randomized trial. Ann Intern Med. 2009:150:255-62.

12. Griffiths F, Lindenmeyer A, Powell J, Lowe P, Thorogood M. Why are health care interventions delivered over the internet? A systematic review of the published literature. J Med Internet Res. 2006;8:e10.

13. Manzoni GM, Pagnini F, Corti S, Molinari E, Castelnuovo G. Internet-based behavioral interventions for obesity: an updated systematic review. Clin Pract Epidemiol Ment Health. 2011;7:19.

14. Riper $\mathrm{H}$, Andersson $\mathrm{G}$, Christensen $\mathrm{H}$, Cuijpers $\mathrm{P}$, Lange A, Eysenbach $\mathrm{G}$ Theme issue on e-mental health: a growing field in Internet research. J Med Internet Res. 2010;12:e74.

15. Arem $\mathrm{H}$, Irwin M. A review of web-based weight loss interventions in adults. Obes Rev. 2011:12:e236-43.

16. Mauriello LM, Sherman KJ, Driskell MM, Prochaska JM. Using interactive behavior change technology to intervene on physical activity and nutrition with adolescents. Adolesc Med State Art Rev. 2007;18:383-99. xiii.

17. Nakade M, Muto T, Hashimoto M, Haruyama Y. Internet-based education program of nutrition as a workplace health promotion tool - a review of the literature. International Congress Series. 2006;1294:135-8.

18. Hamel LM, Robbins LB, Wilbur J. Computer- and web-based interventions to increase preadolescent and adolescent physical activity: a systematic review. J Adv Nurs. 2010:67:251-68.

19. Jahangiry L, Shojaeizadeh D, Najafi M, Mohammad K, Abbasalizad Farhangi M, Montazeri A. 'Red Ruby': an interactive web-based intervention for lifestyle modification on metabolic syndrome: a study protocol for a randomized controlled trial. BMC Public Health. 2014;14:748.

20. Group WGotPCR. Effects of comprehensive lifestyle modification on blood pressure control. JAMA. 2003:289:2083.

21. Schwellinus MP, Patel DN, Nossel CJ, Dreyer M, Whitesman S, Dermen EW. Healthy lifestyle interventions in general practice. Part 6: Lifestyle and metabolic syndrome. SA Fam Pract. 2009;251:177-81.

22. National Institutes of Health. Clinical Guidelines on the Identification, Evaluation, and Treatment of Overweight and Obesity in Adults: The Evidence Report. Obes Res. 1998:6(Suppl 2):51S-2095.

23. WHO. "BMI classification". Global database on body mass index. http://who. int/bmi/index.jsp?introPage=intro_3.html. Accessed May 2013.

24. Bo S, Ciccone G, Baldi C, Benini L, Dusio F, Forastiere G, et al. Effectiveness of a lifestyle intervention on metabolic syndrome: a randomized controlled trial. J Gen Intern Med. 2007;22:1695-703.

25. Despres JP, Lemieux I. Abdominal obesity and metabolic syndrome. Nature. 2006:444:881-7.

26. Jahangiry L, Shojaeizadeh D, Montazeri A, Najafi M, Mohammad K, Yaseri M. Adherence and attrition in a web-based lifestyle intervention for people with metabolic syndrome. Iran J Public Health. 2014;43:1248-58.

27. Kim C-J, Kang S. Development and a pilot test of an Internet-based cardiovascular risk reduction program for Korean male workers with metabolic syndrome. Comput Inform Nurs. 2013;31:157-66.

28. Webster R, Li SC, Sullivan DR, Jayne K, Su SY, Neal B. Effects of Internetbased tailored advice on the use of cholesterol-lowering interventions: arandomized controlled trial. J Med Internet Res. 2010;12:e42.

29. Safran Naimark J, Madar Z, Shahar DR. The impact of a web-based app (eBalance) in promoting healthy lifestyle: randomized controlled trial. J Med Internet Res. 2015;17:e56.

30. Farhangi MA, Jahangiry L, Mirinazhad MM, Shojaeezade D, Montazeri A, Yaghoubi A. A web-based interactive lifestyle modification program improves lipid profile and serum adiponectin concentrations in patients with metabolic syndrome: the "Red Ruby" study. Int J Diabetes Dev Ctries 2015, In press.

31. Carey M, Noble N, Mansfield E, Waller A, Henskens F, Sanson-Fisher R. The role of eHealth in optimizing preventive care in the primary care setting. J Med Internet Res. 2015;17:e126.

32. Bond GE, Burr R, Wolf FM, Price M, McCurry SM, Teri L. The effects of a web-based intervention on the physical outcomes associated with diabetes 
among adults age 60 and older: a randomized trial. Diab Technol Ther 2007:9(1):52-9.

33. Jahangiry L, Shojaeizadeh D, Montazeri A, Najafi M, Mohammad K, Farhangi MA. Modifiable lifestyle risk factors and metabolic syndrome: opportunities for a web-based preventive program. J Res Health Sci. 2014;14:303-7.

34. Enwald HPK, Huotari M-LA. Preventing the obesity epidemic by second generation tailored health communication: an interdisciplinary review. J Med Internet Res. 2010;12:e24.

35. Webb TL, Joseph J, Yardley L, Michie S. Using the internet to promote health behavior change: a systematic review and meta-analysis of the impact of theoretical basis, use of behavior change techniques, and mode of delivery on efficacy. J Med Internet Res. 2010;12:e4.

36. Strecher VJ, McClure JB, Alexander GL, Chakraborty B, Nair VN, Konkel JM, et al. Web-based smoking-cessation programs: results of a randomized trial. Am J Prev Med. 2008;34:373-81

37. D'Agostino RB, Vasan RS, Pencina MJ, Wolf PA, Cobain M, Massaro JM, et al. General cardiovascular risk profile for use in primary care:the Framingham Heart Study. Circulation. 2008;117:743-53.

38. Pearson TA. New tools for coronary risk assessment: what are their advantages and limitations? Circulation. 2002;105:886-92

39. Brouwer W, Kroeze W, Crutzen R, de Nooijer J, de Vries NK, Brug J, et al. Which intervention characteristics are related to more exposure to Internetdelivered healthy lifestyle promotion interventions? A systematic review. $<$ ? show [?A3B2 show \$6\#?]?>J Med Internet Res. 2011:13:e2.

40. Jackson L, Ervin K, Gardner P, Schmitt N. Gender and the Internet: women communicating and men searching. Sex Roles. 2001:44:363-79.

41. Wanner M, Martin-Diener E, Bauer G, Braun-Fahrländer C, Martin BW. Comparison of trial participants and open access users of a web-based physical activity intervention regarding adherence, attrition, and repeated participation. J Med Internet Res. 2010;12:e3.

42. McCully SN, Don BP, Updegraff JA. Using the Internet to help with diet, weight, and physical activity: results from the Health Information National Trends Survey (HINTS). J Med Internet Res. 2013;15:e 148.

43. Deedwania P, Gupta R. Management issues in the metabolic syndrome. JAPI. 2006:54:797-810.

\section{Submit your next manuscript to BioMed Central and take full advantage of:}

- Convenient online submission

- Thorough peer review

- No space constraints or color figure charges

- Immediate publication on acceptance

- Inclusion in PubMed, CAS, Scopus and Google Scholar

- Research which is freely available for redistribution 